INPLASY

PROTOCOL

To cite: Abas et al. Cranial

Polyneuropathy in Bell's Palsy:

A Systematic Review. Inplasy

protocol 202160111. doi:

10.37766/inplasy2021.6.0111

Received: 29 June 2021

Published: 29 June 2021

Corresponding author:

Razif Abas

razifabas@gmail.com

Author Affiliation:

Universiti Putra Malaysia.

Support: No financial support.

Review Stage at time of this submission: Data analysis.

Conflicts of interest:

None declared.

\section{Cranial Polyneuropathy in Bell's Palsy: A Systematic Review}

Abas, R'; Alias, A2; Kamaruzzaman, $\mathrm{MA}^{3}$; Wong, $\mathrm{KH}^{4}$; Hadie, $\mathrm{SNH}^{5}$; Mohd Nor, NF6; Romli, $\mathrm{MH}^{7}$.

Review question / Objective: In the clinical case setting, this study aims to systematically look for symptoms and signs of other cranial nerve(s) involvement in Bell's Palsy, other than the facial nerve.

Condition being studied: Bell's palsy seems to be associated with cranial nerve polyneuritis at least $8 \%$ of the time. The finding that a small but substantial number of Bell's palsy patients have evidence of other cranial nerve involvement is significant. Over a two-year study period, all participants with Bell's palsy who presented to an emergency room were assessed. A total of $\mathbf{5 1}$ patients were involved in the study. Four patients were identified as having extra cranial neuropathies. The current study's merits, however, are its prospective nature and the fact that all of the patients were assessed at the time of their initial presentation by someone who had previous expertise performing neurological examinations. When these inquiries come up empty, it is safe to assume that the cranial polyneuropathy is viral or postinfectious, with a benign prognosis similar to Bell's palsy. Bell's palsy should always be studied further, as polyneuritis cranialis should never be assumed to be a feature of the condition.

INPLASY registration number: This protocol was registered with the International Platform of Registered Systematic Review and Meta-Analysis Protocols (INPLASY) on 29 June 2021 and was last updated on 29 June 2021 (registration number INPLASY202160111).

\section{INTRODUCTION}

Review question / Objective: In the clinical case setting, this study aims to systematically look for symptoms and signs of other cranial nerve(s) involvement in Bell's Palsy, other than the facial nerve.
Condition being studied: Bell's palsy seems to be associated with cranial nerve polyneuritis at least $8 \%$ of the time. The finding that a small but substantial number of Bell's palsy patients have evidence of other cranial nerve involvement is significant. Over a two-year study period, all participants with Bell's palsy who 
presented to an emergency room were assessed. A total of 51 patients were involved in the study. Four patients were identified as having extra cranial neuropathies. The current study's merits, however, are its prospective nature and the fact that all of the patients were assessed at the time of their initial presentation by someone who had previous expertise performing neurological examinations. When these inquiries come up empty, it is safe to assume that the cranial polyneuropathy is viral or postinfectious, with a benign prognosis similar to Bell's palsy. Bell's palsy should always be studied further, as polyneuritis cranialis should never be assumed to be a feature of the condition.

\section{METHODS}

Search strategy: A thorough systematic search was conducted using five electronic databases: CINAHL, Academic Search Complete, MEDLINE, SPORTDiscus, and Scopus, where the initial search is done on 13th February 2020 and updated on 10th May 2021. The development of keywords arose from a discussion among authors and a review of existing literature. The following keywords were used in the research: "Bell palsy" OR "Bell's palsy" OR "Facial Paralysis" OR "Cranial nerve palsy" OR "Facial nerve palsy" OR "Idiopathic nerve palsy" OR "Facial nerve paralysis" OR "Facial palsy" AND "Oculomotor nerve" OR "Trochlear nerve" OR "Abducens nerve" OR "Extraocular muscle" OR "Trigeminal nerve" OR "Mandibular nerve" OR "Olfactory nerve" OR "Optic nerve" OR "Vestibulocochlear nerve" OR "Glossopharyngeal nerve" OR "Vagus nerve" OR "Accessory nerve" OR "Hypoglossal nerve" AND "simultaneous" OR "concurrent" OR "coincid*" OR "concomitant" OR "involve*" OR "associat*" OR "connect" AND "human" OR "People" OR "homo sapiens" OR "patient*" OR "man" OR "men" OR "wom?n" AND NOT "animal*" OR "rabbit*" OR "mice" OR "mouse" OR "rat" OR "rabbit*" OR "monkey*" OR "pig*" OR "primate*" OR "dog*" OR "canine" OR "veterina*". Boolean operators, and other commands such as truncation, wildcards, exact and parenthesis is used whenever appropriate. The reference list of the included study was screened manually as for a manual search. In addition, the cases that were found were checked for the original report. The screening process for eligibility was then performed after relevant citations were chosen.

Participant or population: Patient(s) who diagnosed with Bell's palsy.

Intervention: None.

Comparator: None.

Study designs to be included: Case studies/reports, case series and case control.

Eligibility criteria: The inclusion criteria were (i) any study investigating Bell's palsy, (ii) study in humans, and (iii) involvement of any cranial nerve(s). The final criterion was established by examining the other cranial nerve(s) that were discovered concurrently with Bell's palsy. The exclusion criteria were: (i) animal studies, (ii) investigate nerve(s) other than cranial, (iii) intervention analysis, (iv) full text not available in English, (vi) grey literature (thesis, conference, book), and (vii) no full text available.

Information sources: Before the screening process, duplicates were eliminated. The title was screened for eligibility according to the predetermined requirements by first author (RA), who were then accompanied by independent screening of the abstract and full text by all authors (RA, AA, MAK, WKH, SNHH, CKW, NFMN and MHR). All the full texts were verified among assigned authors. Any differences between the authors' decision were addressed through dialogue until a decision was reached.

Main outcome(s): The optic, oculomotor, trigeminal, vestibulocochlear, glossopharyngeal, vagus, and hypoglossal nerves are among the seven potential cranial nerves associated with Bell's palsy. The trigeminal and vestibulocochlear 
nerves were discovered to be the most involved cranial nerves in Bell's palsy, as shown by advanced laboratory investigations.

Quality assessment / Risk of bias analysis: Screened articles were evaluated for quality using the Joanna Briggs Institute Manual for Evidence Synthesis. Only, case studies/reports, case series and case control were found, therefore, quality evaluation forms for the two designs were selected. The case report evaluation form consists of eight items, while the case series and case control are evaluated using the same evaluation form, which consists of 10 items. For both forms, each item is rated either 'YES', 'NO', 'UNSURE, or 'NOT APPLICABLE'. There is no total score calculated where each item is reported independently. At the end of the appraisal, overall evaluation was performed either to include, exclude or seek further info. RA and MHR administered quality assessments independently, which were then verified through discussion. Sackett's hierarchy levels of evidence, was used in this systematic review to indicate the quality of the evidence. The evidence hierarchy has five levels: Level I (strong) large randomized controlled trial with clear cut result, Level II (moderate) - small RCT, other controlled trials, Level III (moderate) cohort and case-control study, Level IV (weak) - historical cohort and case-control study, and Level V (weak) - case series, studies with no control. Each study is classified into the hierarchy level based on the design. The use of evidence level is valid and useful to guide research application and indicate the trust and credibility of the evidence to be accepted in practice.

Strategy of data synthesis: Included.

Subgroup analysis: Only three of the five Sackett levels of evidence; Levels III, IV, and V, were appropriate for categorizing the final quest in this systematic analysis. At least one cranial nerve involvement has been linked to the onset of Bell's Palsy in thirteen studies. The trigeminal and vestibulocochlear nerves were involved in the majority of the cases, with each having six involvements. The optic and oculomotor nerves, on the other hand, are the least associated, with just one involvement each.

Sensitivity analysis: The narrative review of the articles was used in the final analysis. The research objectives, study design, clinical case setting, findings, cranial nerve(s) involved, and limitation are all extracted from each paper and tabulated into a table. Other cranial nerves than 7th was then identified during its clinical setting, which was based on the presenting symptoms and signs.

Language: English.

\section{Country(ies) involved: Malaysia.}

Keywords: Bell's palsy; cranial nerve; facial nerve; facial paralysis; lower motor neuron palsy, neuroanatomy, clinical anatomy.

\section{Contributions of each author:}

Author 1 - Razif Abas has a major role initiating the idea, involves in searching, screening, disseminating the articles and finalizing the manuscript.

Author 2 - Aspalilah Alias has a role in evaluating the quality of the included studies.

Author 3 - Mohd Amir Kamaruzzaman has a role in preparing and registering the protocol and writing the manuscript.

Author 4 - Wong Kah Hui has a role in verifying data extraction in the table.

Author 5 - Siti Nurma Hanim Hadie has a role in categorizing the articles over the Sackett level of evidence.

Author 6 - Choy Ker Woon synthesizing and charting the data and has a substantial role in critical analysing the manuscript.

Author 7 - Nor Farid Mohd Nor provides a critical feedback on the manuscript.

Author 8 - Muhammad Hibatullah Romli has a role on in critically analysing and writing the manuscript and approving the final manuscript. 